\title{
TNF- $\alpha$ induces calcification of cultured human aortic valve interstitial cells obtained from patients with calcific aortic stenosis: involvement of BMP2
}

\author{
Kazuhiko Seya ${ }^{1}$, Zaiqiang Yu², Mari Chiyoya ${ }^{2}$, Kazuyuki Daitoku², Shigeru Motomura ${ }^{3}$, \\ Tadaatsu Imaizumi ${ }^{1}$, Ikuo Fukuda ${ }^{2}$, Ken-Ichi Furukawa ${ }^{3}$ \\ ${ }^{I}$ Department of Vascular Biology, Hirosaki University Graduate School of Medicine, Japan, ${ }^{2}$ Department of Thoracic \\ Cardiovascular Surgery, Hirosaki University Graduate School of Medicine, Japan, ${ }^{3}$ Department of Pharmacology, \\ Hirosaki University Graduate School of Medicine, Japan
}

Since calcific aortic stenosis (CAS) is characterized by ectopic valve calcification, stenosis of the orifice area, the inhibition of calcification is medically critical. Although tumor necrosis factor- $\alpha$ (TNF- $\alpha$ ) has been detected in calcified valves, the role of TNF- $\alpha$ in valve calcification remains unclear. In this study, we investigated to find whether TNF- $\alpha$ accelerates the calcification of aortic valve by using human aortic valve interstitial cells (HAVICs) obtained from patients with CAS (CAS group) and with aortic regurgitation or aortic dissection having a noncalcified aortic valve (Non-CAS group). HAVICs were cultured in $\alpha$-MEM with $10 \%$ FBS. The medium containing TNF- $\alpha(30 \mathrm{ng} / \mathrm{ml})$ was replenished every $3-4$ days after confluence. The degree of calcification was assessed by Alizarin Red S staining. Gene and protein expression was measured by real-time PCR and western blotting, respectively. TNF- $\alpha$ significantly accelerated the calcification and alkaline phosphatase (ALP) activity of HAVICs from CAS but not Non-CAS group after 14 days of culture. Gene expression of calcigenic markers, ALP, bone morphogenetic protein 2 (BMP2), and distal-less homeobox 5 (Dlx5) were significantly increased after 6 days of TNF- $\alpha$ treatment in CAS but not in Non-CAS group. In HAVICs from CAS group, both SN-50, an inhibitor of NF- $\kappa \mathrm{B}$ p65 subunit translocation into the nucleus, and dorsomorphin, an inhibitor of Smads $1 / 5 / 8$ phosphorylation, strongly inhibited the TNF- $\alpha$-induced calcification. In addition, SN-50 markedly inhibited the BMP2 gene expression and dorsomorphin inhibited both ALP activity and Dlx5 gene expression in HAVICs. These results suggest that HAVICs from CAS group are more sensitive to TNF- $\alpha$, accelerating the calcification of aortic valve via the BMP2-Dlx5-ALP pathway. Meanwhile, TNF- $\alpha$ significantly decreased the gene expression of matrix Gla protein (MGP), an antagonist of BMP2. We further investigated to clarify the role of MGP by regulating MGP gene expression. MGP overexpression strongly inhibited the TNF- $\alpha$-induced HAVICs calcification, while MGP knockdown enhanced HAVICs calcification together with the progression of BMP gene expression. These results suggest that TNF- $\alpha$-induced calcification of HAVICs obtained from CAS patients proceeds via two mechanisms; one is the acceleration of BMP2-Dlx5ALP pathway and another is the negative regulation of MGP gene expression. 